\title{
Diabetic Myonecrosis in a Cystic Fibrosis Patient
}

\author{
Benjamin T Kopp MD, Stephen Kirkby MD, Don Hayes Jr MD, and Kevin M Flanigan MD
}

\begin{abstract}
Cystic fibrosis (CF) related diabetes is an increasingly common comorbidity in CF patients, with scarce data on end-stage complications in the CF population. We report the case of a 32-year-old with poorly controlled diabetes presenting with sub-acute leg pain and focal quadriceps tenderness. Neuromuscular testing and extensive workup revealed diabetic myonecrosis. To our knowledge, this is the first reported case of diabetic myonecrosis in a patient with $\mathrm{CF}$, and highlights the need for pulmonary physicians to recognize this diabetic complication in CF patients, which is associated with a poor long-term prognosis and existing microvascular complications. Key words: cystic fibrosis; diabetes mellitus; leg pain; microvascular; myonecrosis. [Respir Care 2013;58(10):e123-e125. (C) 2013 Daedalus Enterprises]
\end{abstract}

\section{Introduction}

Pharmacologic, nutritional, and screening advancements in cystic fibrosis (CF) have dramatically improved life expectancy. However, due to advancing patient age, the incidence of comorbidities is increasing. In particular, the most common comorbidity, CF-related diabetes mellitus, is present in about $20 \%$ of adolescents and nearly $50 \%$ of adults with CF. ${ }^{1}$ Concerns over plateauing patient life expectancy rates have led to interest in the impact of CF-related diabetes mellitus on CF morbidity and mortality. We present a patient with a rare but important complication of poorly controlled diabetes in a CF patient, one that is potentially under-recognized by respiratory clinicians.

\footnotetext{
The authors are affiliated with the Section of Pediatric Pulmonology, with the exception of Dr Flanigan, who is affiliated with the Division of Neurology, Nationwide Children's Hospital, Ohio State University, Columbus, Ohio.

Dr Kopp presented a version of this paper at the International Conference of the American Thoracic Society, held May 18-23, 2012, in San Francisco, California.

The authors have disclosed no conflicts of interest.

Correspondence: Benjamin T Kopp MD, Section of Pulmonary Medicine, Nationwide Children's Hospital, 700 Children's Drive, Columbus OH 43205. E-mail: Benjamin.Kopp@ NationwideChildrens.org.
}

DOI: $10.4187 /$ respcare.02299

\section{Case Report}

A 32-year-old female with $\mathrm{CF}$, with a baseline $\mathrm{FEV}_{1}$ of $37 \%$ of predicted, poorly controlled CF-related diabetes mellitus (hemoglobin A1C of 11.1), chronic renal insufficiency (with resolved microalbuminuria), and osteoporosis, presented with a 1-month history of fatigue, weight loss, dyspnea, and right thigh pain. The patient had baseline concerns of medication adherence, as indicated by her extremely elevated hemoglobin A1C. At presentation she noted onset of the pain over 1 week, and originally attributed it to muscle strain. Over the ensuing month the pain became severe, and she noted increasingly exquisite tenderness to palpation of her thigh. She denied any weakness or functional loss, and she was able to continue working.

She was admitted to the hospital for evaluation. She was afebrile, and her examination showed no skin or nail bed abnormalities. Her cranial nerve examination was normal, without cranial neuropathies. She was thinly muscled throughout. She had marked tenderness to palpation of the right anterior and superior thigh muscles, with a thigh circumference that was $5 \mathrm{~mm}$ larger on the right. Isolated muscle testing showed 5/5 strength in all groups except at shoulder abduction (4+ bilaterally), hip flexion (4+ on the right, and 5 on the left), knee extension $(4+$ on the right, 5 on the left), and great toes abduction (4/5 bilaterally). Pin-prick sensation was reduced in a stocking fashion, normalizing at the mid-calf, and was notably normal across the thighs bilaterally. Vibration was diminished in a stocking-glove fashion, and ankle jerks were absent bilaterally. 


\section{Diabetic Myonecrosis in a Cystic Fibrosis Patient}

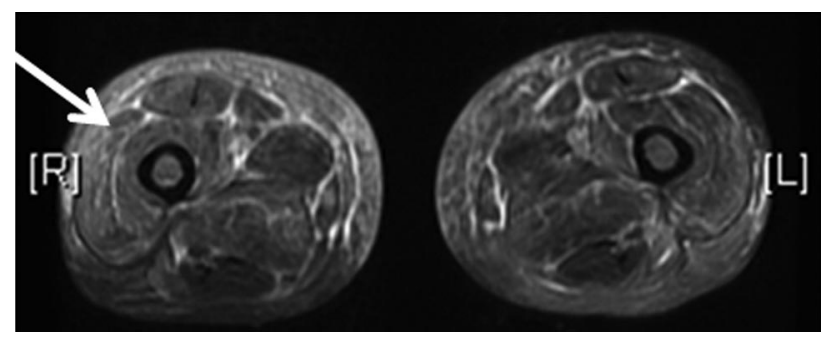

Fig. 1. T2-weighted axial magnetic resonance imaging of the anterior thigh shows diffuse subcutaneous tissue edema and diffusely abnormal signal intensity within the thigh musculature. The arrow points to highest signal within the vastus lateralis.

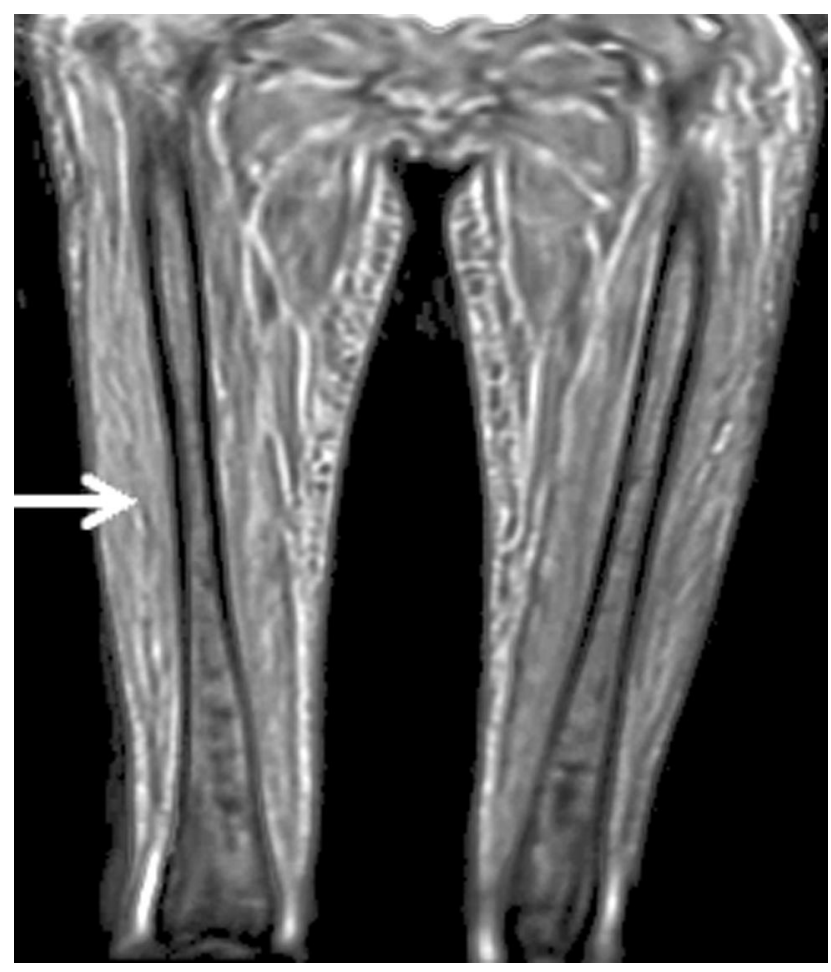

Fig. 2. Coronal magnetic resonance imaging of the thigh shows diffuse abnormal signal intensity, greatest in the right vastus lateralis (arrow).

Plain films of the right leg revealed no acute fractures. A bone scan revealed no occult infection or injury, but increased radiotracer uptake at the patella. An initial knee magnetic resonance imaging (MRI) demonstrated a small right knee joint effusion, but an MRI of the thighs demonstrated abnormal enhancing T2 signal within the anterior thigh musculature (Figs. 1 and 2). At the time of admission her serum creatine kinase was normal, at $69 \mathrm{U} / \mathrm{L}$ (normal range 37-289 U/L), rheumatoid factor was $<7$ IU/ $\mathrm{mL}$, lactate dehydrogenase was $538 \mathrm{U} / \mathrm{L}$, and white blood cell count was $10.7 \times 10^{3} / \mathrm{mL}$. Her erythrocyte sedimentation rate was elevated, at $53 \mathrm{~mm} / \mathrm{h}$.
Given her clinical course, including a history of uncontrolled diabetes with microvascular complications, the characteristic myonecrosis indicated by examination and MRI, and the absence of laboratory evidence for another infectious or rheumatologic cause, the diagnosis was determined to be diabetic myonecrosis. Along with treatment with intravenous antibiotics and chest physiotherapy for a pulmonary exacerbation, she was placed on bed rest, with temporary avoidance of physical activity. She required narcotics for pain control (oxycodone twice a day), and was encouraged to maintain strict glycemic control. Her acute symptoms resolved over approximately 2 weeks, but she has had chronic leg pain requiring long-term painclinic follow-up. Her distal lower extremity sensory abnormalities persist due to diabetic neuropathy, and are treated with gabapentin, and she has no known ophthalmologic abnormalities. Additionally, her glycemic control has only modestly improved, to a hemoglobin A1C of 10.1, despite physician interventions.

\section{Discussion}

First reported in $1965,{ }^{2}$ diabetic myonecrosis has since been reported in approximately 100 cases worldwide, but to our knowledge this is the first case associated with CF-related diabetes mellitus. It is essential to recognize myonecrosis, a rare complication of uncontrolled diabetes, as an ominous sign of worsening diabetes, because many patients die within 5 years of onset, due to existing or worsening microvascular complications. ${ }^{3}$ Diabetic myonecrosis is caused by muscle infarction, leading to acute muscle pain in the absence of trauma, usually located in the quadriceps. Pain is typically unilateral, but is bilateral in up to $8 \%$ of cases. ${ }^{4}$ The pathogenesis is still unknown, with some groups advocating diabetic microangiopathy, while others speculate on an association with hypercoagulable factors. ${ }^{5}$ Serum markers, including creatine kinase, are normal in approximately $50 \%$ of cases. ${ }^{4}$ Radiologic imaging characteristics of the diagnosis include MRI with increased T2-weighted signal in the affected muscle secondary to edema and to inflammatory changes from infarction. ${ }^{6}$ Bedside ultrasound can be a useful imaging technique to rule out other causes of leg pain and to detail muscle architecture. ${ }^{7}$ Needle electromyography is often done clinically if the diagnosis is uncertain, but the results can be variable and nonspecific, limiting the diagnostic use in this setting, unless the diagnosis is in doubt. Muscle biopsy may help to exclude other syndromes, such as focal nodular myositis, which may present with similar radiographic and clinical features, ${ }^{8}$ but in the appropriate clinical setting it is not necessary for diagnosis, and is not routinely recommended, due to associated complications, such as delayed wound healing. ${ }^{9}$ Other diagnostic considerations to exclude in making the diagnosis include infec- 


\section{Diabetic Myonecrosis in a Cystic Fibrosis Patient}

tions, such as pyomyositis and necrotizing fasciitis, tumor, thrombosis, dermatomyositis, and diabetic lumbosacral radiculoplexus neuropathy, as several of these can be acutely life-threatening and could require a tissue biopsy.

Treatment of diabetic myonecrosis is aimed at improving glycemic levels, along with control of generally exquisite pain through non-steroidal anti-inflammatories and opioid analgesics. Strict bed rest helps improve the selflimiting nature of diabetic myonecrosis, and physical therapy has been reported to prolong symptoms in about $14 \%$ of cases. Recurrences occur in nearly half of the cases, with a new muscle affected in $82 \%$ of the recurrences. ${ }^{9}$ The use of anti-coagulants has been suggested, but not proven to be of benefit. ${ }^{10}$ Although short-term prognosis for patients affected with diabetic myonecrosis is good, the 5-year survival rate is poor, due to existing microvascular complications of uncontrolled diabetes. ${ }^{3}$ Additionally, females with CF-related diabetes mellitus have a decreased overall survival rate, compared to males, even in the absence of diabetic myonecrosis, putting our patient at greater risk. ${ }^{11}$

Macrovascular complications of CF-related diabetes mellitus are currently rare in $\mathrm{CF}$, presumably due to low cholesterol levels from $\mathrm{CF}$ transmembrane conductance regulator ion channel defects or fat malabsorption. However, microvascular defects are common in patients with diabetic symptoms of $>10$ years duration, ${ }^{12}$ and upwards of $27 \%$ of $\mathrm{CF}$ patients on insulin have been reported to have other microvascular complications, such as diabetic retinopathy. ${ }^{13}$ It is therefore essential that respiratory caregivers recognize the clinical syndrome of diabetic myonecrosis, understand that it may signal impending microvascular catastrophe, and to be alert for this diagnosis in patients with existing microvascular complications. The recommendation is to start screening for CF-related diabetes mellitus at age 10, with an annual oral glucose tolerance test to help identify patients early and prevent development of microvascular complications. ${ }^{14}$ Quarterly hemoglobin A1C measurements, with a goal of $<7 \%$, are recommended to prevent microvascular complications in CF. ${ }^{14}$ Annual neurologic, ophthalmologic, and proteinuria screenings are also recommended, starting 5 years after the diagnosis of CF-related diabetes mellitus. Although our patient had a history of resolved microalbuminuria, there is a high incidence (21\%) in patients with CF-related diabetes mellitus, compared to type 1 diabetics, which may reflect other disease and medication factors in CF. ${ }^{15}$

In summary, diabetic myonecrosis is a rare, but ominous, complication of uncontrolled diabetes, and respira- tory physicians need to be aware of it in patients with poorly controlled CF-related diabetes mellitus who present with leg pain.

\section{ACKNOWLEDGMENTS}

We thank Fred Long MD, Julie O'Donovan MD, and Brent Adler MD for radiologic input.

\section{REFERENCES}

1. Moran A, Dunitz J, Nathan B, Saeed A, Holme B, Thomas W. Cystic fibrosis-related diabetes: current trends in prevalence, incidence, and mortality. Diabetes Care 2009;32(9):1626-1631.

2. Angervall LSB. Tumoriform focal muscular degeneration in two diabetic patients. Diabetologia 1965;1:39-42.

3. Rocca PV, Alloway JA, Nashel DJ. Diabetic muscular infarction. Semin Arthritis Rheum 1993;22(4):280-287.

4. Trujillo-Santos AJ. Diabetic muscle infarction: an underdiagnosed complication of long-standing diabetes. Diabetes Care 2003;26(1): 211-215.

5. Palmer GW, Greco TP. Diabetic thigh muscle infarction in association with antiphospholipid antibodies. Semin Arthritis Rheum 2001; 30(4):272-280.

6. Jelinek JS, Murphey MD, Aboulafia AJ, Dussault RG, Kaplan PA, Snearly WN. Muscle infarction in patients with diabetes mellitus: MR imaging findings. Radiology 1999;211(1):241-247.

7. Nagdev A, Murphy M, Sisson C. Bedside ultrasound for the detection of diabetic myonecrosis. Am J Emerg Med 2008;26(8):969 e963-964.

8. Jelinek J, Kransdorf MJ. MR imaging of soft-tissue masses. Masslike lesions that simulate neoplasms. Magn Reson Imaging Clin N Am 1995;3(4):727-741.

9. Chester CS, Banker BQ. Focal infarction of muscle in diabetics. Diabetes Care 1986;9(6):623-630.

10. Bjornskov EK, Carry MR, Katz FH, Lefkowitz J, Ringel SP. Diabetic muscle infarction: a new perspective on pathogenesis and management. Neuromuscul Disord 1995;5(1):39-45.

11. Milla CE, Billings J, Moran A. Diabetes is associated with dramatically decreased survival in female but not male subjects with cystic fibrosis. Diabetes Care 2005;28(9):2141-2144.

12. Schwarzenberg SJ, Thomas W, Olsen TW, Grover T, Walk D, Milla $\mathrm{C}$, et al. Microvascular complications in cystic fibrosis-related diabetes. Diabetes Care 2007;30(5):1056-1061.

13. Andersen HU, Lanng S, Pressler T, Laugesen CS, Mathiesen ER. Cystic fibrosis-related diabetes: the presence of microvascular diabetes complications. Diabetes Care 2006;29(12):2660-2663.

14. Moran A, Brunzell C, Cohen RC, Katz M, Marshall BC, Onady G, et al. Clinical care guidelines for cystic fibrosis-related diabetes. A position statement of the American Diabetes Association and a clinical practice guideline of the Cystic Fibrosis Foundation, endorsed by the Pediatric Endocrine Society. Diabetes Care 2010; 33(12):2697-2708.

15. van den Berg JM, Morton AM, Kok SW, Pijl H, Conway SP, Heijerman HG. Microvascular complications in patients with cystic fibrosis-related diabetes (CFRD). J Cyst Fibros 2008;7(6):515-519. 